\title{
Paraganglioma of the left atrium
}

\author{
K. M. John Chan, AFRCS, David Pontefract, MRCS, Richard Andrews, MRCP, and Surendra K. Naik, PhD, \\ FRCS (CTh), Nottingham, United Kingdom
}

$\mathrm{P}$ aragangliomas are rare tumors of neural crest origin. They commonly occur in the adrenal medulla, where they are referred to as pheochromocytomas. Other common sites of origin of paragangliomas include the carotid body in the neck and the glomus jugulare of the middle ear. Paragangliomas arising from the heart are extremely uncommon, and only a few cases have been reported.

\section{Clinical Summary}

A 55-year-old man with chest tightness and shortness of breath was admitted to the hospital. Results of his physical examination were unremarkable. An electrocardiogram revealed normal sinus rhythm and no ischemic changes, and a chest radiograph showed no abnormalities. Stress electrocardiography had to be abandoned because the patient felt lightheaded and short of breath. Cardiac catheterization revealed normal coronary arteries and good left ventricular function. An intracardiac mass was seen in the left atri-

From the Department of Cardiothoracic Surgery, Nottingham City Hospital, Nottingham, United Kingdom.

Received for publication March 5, 2001; accepted for publication March 22, 2001.

Address for reprints: Mr S. K. Naik, Consultant Cardiac Surgeon, Nottingham City Hospital, Hucknall Road, Nottingham NG5 3PB, United Kingdom.

J Thorac Cardiovasc Surg 2001;122:1032-3

Copyright (C) 2001 by The American Association for Thoracic Surgery

$0022-5223 / 2001 \$ 35.00+0 \quad \mathbf{1 2 / 5 4 / 1 1 5 9 2 8}$

doi: $10.1067 / \mathrm{mtc} .2001 .115928$ um, with a small collateral vessel from the circumflex artery and a large collateral vessel from the proximal right coronary artery feeding it (Figure 1). Transesophageal echocardiography confirmed the left atrial mass, which measured approximately $3.5 \times 3$ $\mathrm{cm}$. It was discrete from the left atrial appendage and mitral valve and did not appear to obstruct the left pulmonary veins. Computed tomography interestingly failed to demonstrate the intracardiac mass. No masses were seen elsewhere.

The patient underwent an operation. During initial warm antegrade and then continuous warm retrograde cardioplegia, the aorta and pulmonary artery were transected in the transverse sinus and reflected forward to expose the tumor. The tumor was excised with a fringe of atrial tissue (Figure 2). A bovine pericardial patch was used to reconstruct the defect. The patient was weaned off bypass without difficulty.

The tumor was $4 \times 2.7 \times 2 \mathrm{~cm}$ and weighed $12.5 \mathrm{~g}$. It had a rubbery consistency and showed a yellowish rim with pale and hemorrhagic areas centrally on sectioning. The tumor was composed of nests of epithelioid cells with eosinophilic cytoplasm and ovoid nuclei with conspicuous nucleoli. The nuclei were overall mildly pleomorphic, with occasional bizarre and enlarged forms and scattered mitoses. The appearances were those of a paraganglioma. The patient remains well and asymptomatic 6 months after the operation.

\section{Discussion}

Paragangliomas are rare tumors arising from neural crest cells. They occur mainly in adults aged between 30 and 60 years and are more common in women. The common site of origin of paragangliomas is the adrenal medulla. There they are referred to as pheochromocy- 


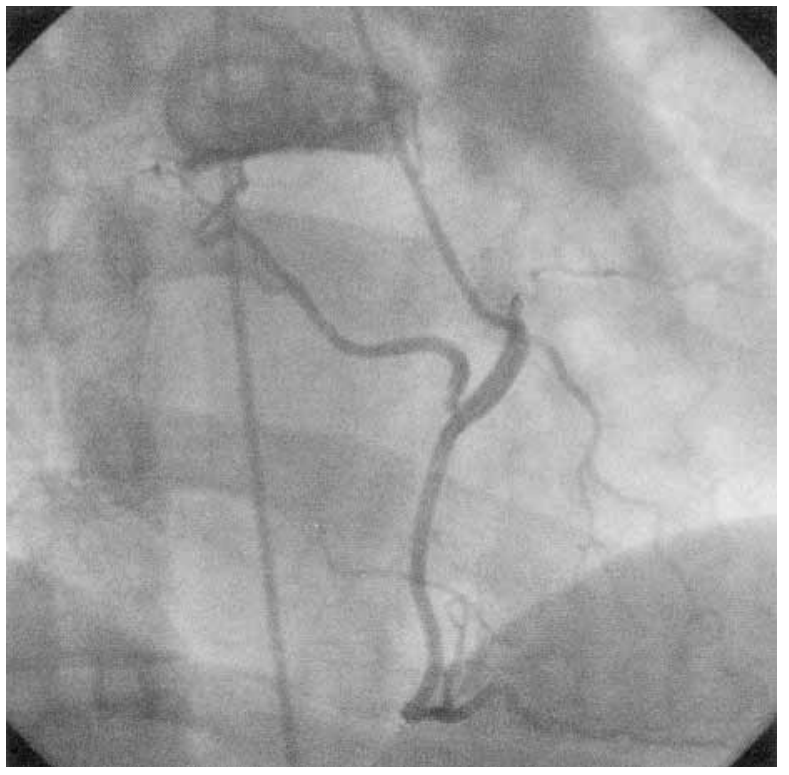

Figure 1. Coronary angiogram, left anterior oblique view, showing the right coronary artery giving off a large collateral vessel, which is feeding the tumor.

tomas. ${ }^{1}$ Paragangliomas arising from the adrenal medulla and the aorticosympathetic ganglia are innervated by the sympathetic nervous system and secrete catecholamines. In contrast, paragangliomas arising from elsewhere generally do not secrete catecholamines and are innervated by the parasympathetic nervous system. Their sites of origin include the carotid body, the jugular ganglion of the vagus, the middle ear, and the ciliary glomus in the orbit. ${ }^{1}$

Paragangliomas originating from the heart are extremely uncommon. Only a few cases have been reported, and most of these were incidental findings at autopsy.,3 The treatment of paragangliomas arising from the heart is similar to that of all other cardiac tumors: they should be completely excised whenever possible. ${ }^{2-4}$ Most patients have symptoms necessitating removal of the tumor, as was the case with our patient, who had limiting angina. Although most paragangliomas are benign, local invasion can occur, and metastases have been reported in up to $10 \%$ of cases. ${ }^{1,4}$ It is therefore important that the tumor be completely excised. There is no correlation between the histologic characteristics of the tumor and its malignant potential. Chemotherapy has not been found to be useful, and results with radiotherapy have been variable.

It is unclear from the literature what follow-up investigations, if any, should be done to identify recurrences. It would seem sensi-

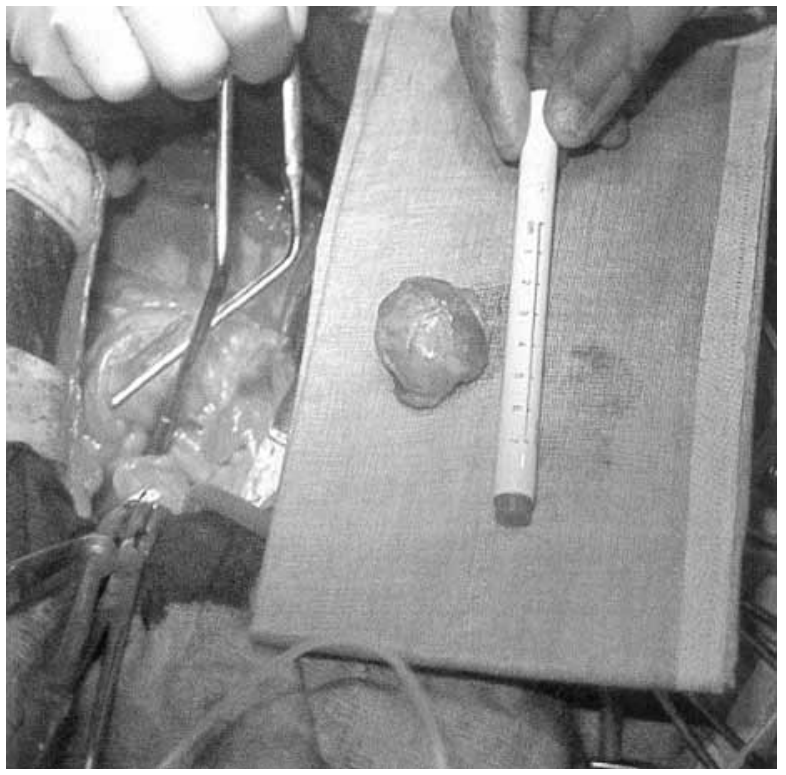

Figure 2. Operative photograph showing the excised tumor from the left atrium.

ble to repeat echocardiography at regular intervals, but metastases can occur anywhere in the body. ${ }^{2}$ The literature also offers no consensus on the treatment for recurrences. The long-term outlook for patients with excised cardiac paragangliomas is unknown. However, survival of up to 14 years has been reported in patients with excised mediastinal paragangliomas. ${ }^{5}$

\section{References}

1. DeLellis RA. The endocrine system. In: Contran RS, Kumar V, Robbins SL, editors. Robbins pathologic basis of disease. 4th ed. Philadelphia: WB Saunders; 1989. p. 1267-8.

2. Gopalakrishnan R, Ticzon A, Cruz P, Kennedy FB, Duffy FC, Barmada B, et al. Cardiac paraganglioma (chemodectoma): a case report and review of the literature. J Thorac Cardiovasc Surg. 1978;76:183-9.

3. Cane ME, Berrizbeitia LD, Yang SS, Mahapatro D, McGrath LB. Paraganglioma of the interatrial septum. Ann Thorac Surg. 1996;61:1845-7.

4. Olson JL, Salyer WR. Mediastinal paragangliomas (aortic body tumour): a report of four cases and a review of the literature. Cancer. 1978;41:2405-14

5. Pachter MR. Mediastinal nonchromaffin paraganglioma. J Thorac Cardiovasc Surg. 1963;45:152-60. 